\title{
Caso fulminante de rickettsiosis (Rickettsia rickettsii) en una lactante del sureste de México
}

\author{
Fatal case of rickettsiosis in a toddler from southeastern Mexico
}

\author{
Dr. César Lugo-Caballero ${ }^{a}$, Dra. Karla Dzul-Rosado ${ }^{a}$,Dra. Georgina Rodríguez-Moreno ${ }^{b}$, \\ Q. F. B. Raúl Tello-Martín ,Q. F. B. Karina López-Ávila y Dr. Jorge Zavala-Castro ${ }^{a}$
}

\begin{abstract}
RESUMEN
La fiebremanchada delas Montañas Rocosas es una enfermedad ocasionada por Rickettsia rickettsii, una bacteria transmitida por garrapatas infectadas, y que se caracteriza por fiebre, exantema, artralgias y mialgias, aunque, ocasionalmente, su presentación es inespecífica. Debido a que su evolución asemeja otras enfermedades exantemáticas, como dengue o chikungunya, su diagnóstico no es de primera intención, a pesar de que países como México tienen las características ecológicas y socioeconómicas propicias para su transmisión, con índices de mortalidad hasta de $30 \%$ en pacientes pediátricos. Esta elevada mortalidad se asocia a diagnósticos y terapia retrasados debido al desconocimiento médico acerca de la enfermedad, lo que propicia la aparición de formas atípicas y fulminantes de fiebre manchada de las Montañas Rocosas.

El objetivo del presente trabajo es describir un caso clínico fulminante de fiebre manchada de las Montañas Rocosas para que sea considerada en el diagnóstico diferencial, lo cual impactaría directamente en los índices de mortalidad.

Palabras clave: rickettsiosis, fiebre maculosa de las Montañas Rocosas, pediatría, enfermedades transmitidas por garrapatas.
\end{abstract}

\begin{abstract}
Rocky Mountain spotted fever is a disease caused by Rickettsia rickettsii, a bacteria transmitted by infected ticks. It is characterized by fever, exanthema, arthralgias and myalgias; but sometimes its clinical presentation is non specific. Due to its similarities with other exanthematic diseases like dengue or chikungunya, Rocky Mountain spotted fever is not a first line diagnosis, even though countries like Mexico show the ecologic and socioeconomic characteristics that favor its transmission, with a $30 \%$ mortality rate among pediatric patients. This mortality rate has been associated to a delayed diagnosis
\end{abstract}

a. Laboratorio de Enfermedades Emergentes y Reemergentes, Centro de Investigaciones Regionales "Dr. Hideyo Noguchi" (CIR), Universidad Autónoma de Yucatán, Mérida, Yucatán, México.

b. Unidad Médica de Alta Especialidad, Centro Médico Nacional "Ignacio García Téllez, IMSS".

Correspondencia:

Dr. César Lugo Caballero, cesar.lugo@correo.uady.mx

Financiamiento: Ninguno.

Conflicto de intereses: Ninguno que declarar.

Recibido: 25-1-2016.

Aceptado: 1-8-2016 and therapy, due to a poor knowledge among physicians regarding this disease; this favors the occurrence of atypical and fulminant cases.

The objective of this work is to describe a fulminant case of Rocky Mountain spotted fever, expecting that this disease could be later considered among the differential diagnosis which could directly impact its mortality rate.

Key words: rickettsia infections, Rocky Mountain spotted fever, pediatrics, tick-borne disease.

http:/ /dx.doi.org/10.5546/aap.2017.e5

\section{INTRODUCCIÓN}

La fiebre de las Montañas Rocosas (RMSF) es una enfermedad potencialmente mortal muy distribuida en el continente americano, que es causada por Rickettsia rickettsii, una bacteria intracelular obligada que se transmite a los seres humanos por diversas especies de garrapatas infectadas. ${ }^{1}$ El cuadro clínico de esta enfermedad deriva de la infección del endotelio vascular, que produce una vasculitis evidenciable como exantema petequial, asociada a fiebre y dolor en el sistema músculo-esquelético. ${ }^{1,2}$ Diversos estudios resaltan la importancia que tiene la falta de difusión y conocimiento de esta patología por el personal de salud, que conlleva un retraso en el diagnóstico y tratamiento temprano, el cual puede explicar las elevadas cifras de mortalidad debidas, principalmente, a sepsis y falla orgánica múltiple. , $^{13,4}$

En México, los últimos reportes del sistema de vigilancia epidemiológica muestran una incidencia de 0,8 casos por 100000 habitantes, distribuidos en el $87 \%$ de los estados del país. ${ }^{5}$ Esta cifra se ha incrementado en estados del norte, como Sonora, donde se ha reportado mayor incidencia de casos en escolares (de 5 a 9 años de edad) con una mortalidad de $30 \%$, cifra superior a la reportada por otros países endémicos, como Estados Unidos (11,7\%), ${ }^{3,6}$

Obedeciendo a la necesidad de difundir el conocimiento de esta enfermedad entre la comunidad médica, se presenta un caso clínico fulminante ocasionado por $R$. rickettsii en el sureste de México, cuya rápida evolución en 
lactantes no había sido observada. El objetivo es que la RMSF sea considerada en el diagnóstico diferencial para evitar así complicaciones o un desenlace fatal y favorecer el tratamiento oportuno.

\section{CASO CLÍNICO}

Paciente femenino de 1 año y 11 meses de edad previamente sana, residente del área conurbana de la ciudad, con antecedentes de vida en hacinamiento, que acudió al hospital en mayo. Los padres refirieron que, desde el nacimiento, había tenido contacto estrecho con animales, específicamente perros y gatos, los cuales no recibían tratamiento preventivo contra garrapatas y eran parasitados por estas con frecuencia.

Inició el padecimiento con hipertermia aguda, que persistió 3 días, de tipo intermitente con picos de hasta $38,8^{\circ} \mathrm{C}$, acompañada de exantema macular generalizado. Al tercer día, se agregó dolor abdominal difuso tipo cólico, que, por su intensidad, ameritó el ingreso al segundo nivel de atención médica para estudio. Durante las primeras horas de su estancia, presentó crisis convulsivas parciales complejas, secundariamente generalizadas por lapso de 40 minutos, por lo que se aseguró la vía aérea mediante intubación endotraqueal y se inició impregnación con difenilhidantoína, diazepam y cisatracurio.

FIGURA 1. Lesiones exantemáticas en la extremidad inferior

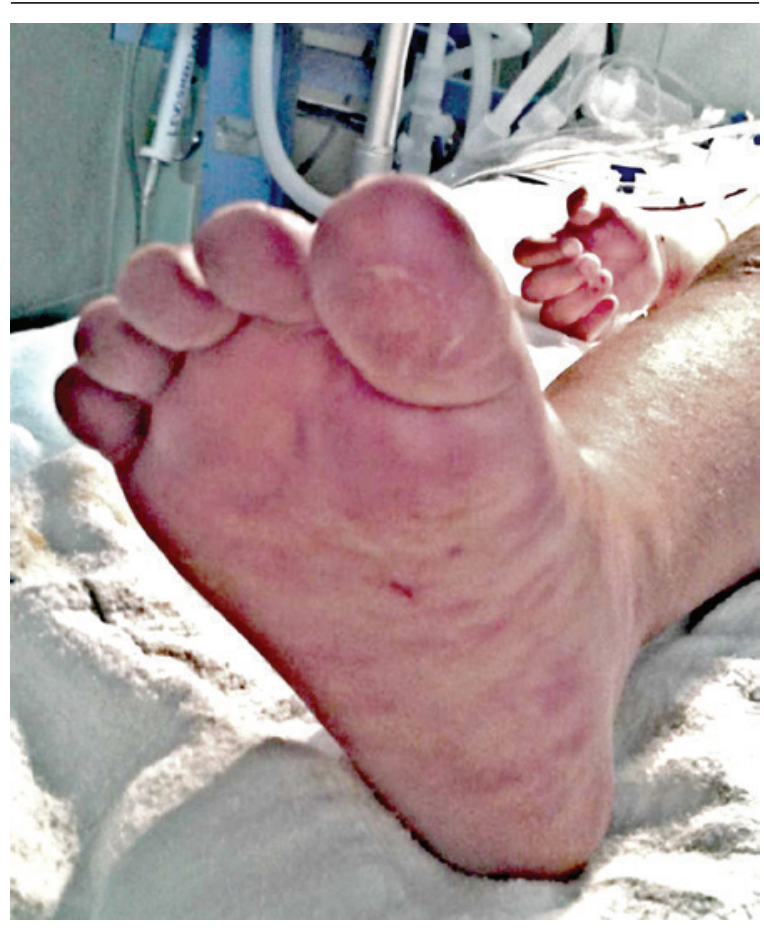

Asimismo, se instaló un catéter venoso central y se trasladó a la paciente a la Unidad de Cuidados Intensivos Pediátricos (UCIP).

La paciente ingresó a la UCIP en malas condiciones generales, con datos de choque séptico y bajo gasto cardíaco manifestado por hipotensión arterial debajo del percentil 5 para su edad, taquicardia, anuria, hipoperfusión distal, llenado capilar retardado, pulsos centrales débiles y pulsos periféricos no palpables. La paciente también presentó lesiones petequiales y purpúricas en el abdomen y en las extremidades tanto superiores como inferiores e isquemia distal en los dedos, datos que reflejaron un proceso vasculítico (Figura 1).

Durante su estancia, se mantuvo la ventilación mecánica controlada por sedación y analgesia con fentanilo/midazolam, esquema de meropenem/ vancomicina ajustado a daño renal, carga de soluciones cristaloides a $20 \mathrm{ml} / \mathrm{kg} /$ día, corrección aguda de bicarbonato a déficit de bases, apoyo inotrópico con milrinona/dobutamina / levisomendan, norepinefrina como vasopresor sistémico, hidrocortisona en infusión para el manejo de choque refractario y heparina en infusión para el manejo de coagulación intravascular diseminada (CID). Se obtuvo gasometría, que mostró acidosis metabólica descompensada, con saturación venosa de $49 \%$ y una lectura de presión venosa central (PVC) de $14 \mathrm{cmH}_{2} \mathrm{O}$. Los análisis paraclínicos dejaron ver una falla orgánica inminente, con compromiso renal y hepático, así como marcadas leucocitosis y trombocitopenia (Tabla 1), por lo que se administraron $10 \mathrm{ml} / \mathrm{kg}$ de plasma fresco y 2 concentrados plaquetarios.

Debido a los antecedentes y al estado clínico de la paciente, se solicitó el diagnóstico de rickettsiosis a nuestro Instituto. Este se llevó a cabo mediante inmunofluorescencia indirecta (IFI) positiva 1:128 para antígenos de $R$. rickettsii circulantes en nuestro medio, en conjunto con la amplificación y secuenciación del gen OmpB, que resultó con homología del 98\% a $R$. rickettsii, así como amplificación anidada del gen $17 k D a$ (Tabla 1). Poco después de las 24 horas de su ingreso y antes de 6 horas en la UCIP, la paciente presentó datos de choque séptico secundario a falla orgánica múltiple, CID y paro cardiorrespiratorio que no respondió a maniobras avanzadas de reanimación y falleció. El diagnóstico serológico fue obtenido horas antes de fallecer la paciente, mientras que el molecular fue obtenido post mórtem. 


\section{DISCUSIÓN}

El espectro clínico de la RMSF abarca desde una enfermedad exantemática febril benigna hasta una entidad de manifestaciones clínicas graves potencialmente fatales..$^{6-9}$ Aunque, en nuestro medio, solamente se ha documentado un caso fatal de RMSF en un paciente escolar, ${ }^{8}$ este número podría ser mayor, debido a un retraso o ausencia de diagnóstico. ${ }^{10}$

En México, el bajo nivel socioeconómico y el contacto estrecho con animales que tienen un mal control de ectoparásitos son las principales condiciones asociadas a RMSF. ${ }^{11}$ De acuerdo con la literatura, las garrapatas $R$. sanguineus son los vectores responsables de la transmisión de $R$. rickettsii $\mathrm{y}$, aunque se ha sugerido que las zarigüeyas funcionan como reservorio, diversos estudios indican que las garrapatas mismas son el reservorio principal de esta bacteria, puesto que la transmisión vertical es el mecanismo más importante para su circulación. ${ }^{12,13}$ Los perros no juegan un papel importante en el ciclo de estas bacterias, pero al ser sensibles a la enfermedad, actúan como centinelas y permiten prevenir la aparición de brotes en poblaciones humanas. ${ }^{12,13}$
No obstante, tienen un papel amplificador como consecuencia de la coalimentación de garrapatas infectadas, que ocasiona la transmisión horizontal de bacterias a las no infectadas, y también ayudan al contacto de garrapatas infectadas con humanos, particularmente niños, por lo que es importante emprender acciones que eviten su infestación. ${ }^{12,13}$

La paciente presentó exantema macular, fiebre de difícil control, dolor abdominal intenso y crisis convulsivas que ameritaron su ingreso al hospital. El exantema aparece entre el día 3 y 5 posinfección en un $80 \%$ de los pacientes, con características eritematosas que evolucionan a purpúricas en los días subsecuentes, evolución que no pudo ser observada en este caso. ${ }^{1,2}$ No existen antecedentes de picadura por garrapatas, lo que concuerda con diversos trabajos que señalan que es referida por un $50 \%$ de los pacientes. ${ }^{2}$ Aunque esta presentación es suficiente para un diagnóstico basado en sospecha, la RMSF no es considerada como una posibilidad inicial. ${ }^{1}$

De acuerdo con las guías internacionales actuales, para la confirmación serológica, se considera la IFI como el estándar de oro, a partir del título 1:64, que tiene validez diagnóstica

TABLA 1. Resultados de laboratorio

\begin{tabular}{|c|c|c|}
\hline Parámetro & Paciente & Referencia \\
\hline Glucosa & $41 \mathrm{mg} / \mathrm{dl}$ & $70-105 \mathrm{mg} / \mathrm{dl}$ \\
\hline Urea & $82 \mathrm{mg} / \mathrm{dl}$ & $7-18 \mathrm{mg} / \mathrm{d}$ \\
\hline Creatinina & $2,9 \mathrm{mg} / \mathrm{dl}$ & $0,5-1,4 \mathrm{mg} / \mathrm{dl}$ \\
\hline $\begin{array}{l}\text { Nitrógeno ureico en } \\
\text { sangre (BUN) }\end{array}$ & $16 \mathrm{mg} / \mathrm{dl}$ & $4,9-22,6 \mathrm{mg} / \mathrm{dl}$ \\
\hline $\mathrm{Na}$ & $137 \mathrm{mEq} / \mathrm{L}$ & $136-146 \mathrm{mEq} / \mathrm{L}$ \\
\hline $\mathrm{Cl}$ & $97 \mathrm{mEq} / \mathrm{L}$ & $102-109 \mathrm{mEq} / \mathrm{L}$ \\
\hline $\begin{array}{l}\mathrm{K} \\
\mathrm{Mg}\end{array}$ & $\begin{array}{c}5,1 \mathrm{mEq} / \mathrm{L} \\
3 \mathrm{mEg} / \mathrm{L}\end{array}$ & $\begin{array}{c}3,5-5 \mathrm{mEg} / \mathrm{L} \\
1,5-2,3 \mathrm{mE} / \mathrm{L}\end{array}$ \\
\hline Hemoglobina & $7,5 \mathrm{~g} / \mathrm{dl}$ & $11,5-16,5 \mathrm{~g} / \mathrm{dl}$ \\
\hline Hematocrito & $21,9 \%$ & $37 \%-54 \%$ \\
\hline Plaquetas $/ \mathrm{mm}^{3}$ & 14000 & $150000-450000$ \\
\hline Leucocitos totales $/ \mathrm{mm}^{3}$ & 19000 & $5000-10000$ \\
\hline Tiempo de protrombina & 25 segundos & $25-35$ segundos \\
\hline Tiempo de tromboplastina & No cuantificable & 11-13 segundos \\
\hline $\begin{array}{l}\text { Aspartato amino- } \\
\text { transferasa (TGO) }\end{array}$ & 221 UIL & $10-42 \mathrm{UI} / \mathrm{L}$ \\
\hline $\begin{array}{l}\text { Alanino amino-transferasa } \\
\text { (TGP) }\end{array}$ & 869 UI/ & $10-40 \mathrm{UI} / \mathrm{L}$ \\
\hline $\begin{array}{l}\text { Lactato deshidrogenasa } \\
\text { (LDH) }\end{array}$ & $2055 \mathrm{UI} / \mathrm{L}$ & 0-480 UI/L \\
\hline Albúmina & $2,1 \mathrm{~g} / \mathrm{dl}$ & $3,5-5 \mathrm{~g} / \mathrm{dl}$ \\
\hline Bilirrubina indirecta & $1,5 \mathrm{mg} / \mathrm{dl}$ & $0,2-1 \mathrm{mg} / \mathrm{dl}$ \\
\hline IFI IgM R. rickettsii & $1: 128$ & $1: 64$ \\
\hline IFI lgM R. tiphy & $1: 64$ & $1: 64$ \\
\hline PCR anidado $17 \mathrm{kDa}$ & + & + \\
\hline PCR anidado $O m p B$ & + & + \\
\hline Número taxonómico & 1337396 & NA \\
\hline
\end{tabular}

IFI: inmunofluorescencia indirecta; PCR: reacción en cadena de la polimerasa. 
al demostrar, en tomas diferentes, una seroconversión de $\operatorname{IgM}$ a $\operatorname{IgG}$ a las 4 semanas de inicio del cuadro clínico. Para el diagnóstico molecular, genes como $17 k D a, O m p A$ u OmpB se amplifican mediante reacción en cadena de la polimerasa con secuenciación del producto para identificar la especie patógena. ${ }^{1}$ En este caso, se obtuvo un diagnóstico serológico (IFI) complementado con una prueba molecular a 24 horas del ingreso (72 $\mathrm{h}$ de haber iniciado el cuadro clínico); sin embargo, la evolución súbita no permitió comenzar el tratamiento con doxiciclina. ${ }^{1,14}$ Cabe destacar que existen estudios que confieren valor pronóstico a los estudios paraclínicos en conjunto con los datos clínicos, en función del daño orgánico que reflejan; en este caso, la leucocitosis, datos de compromiso cardíaco y renal, edad pediátrica, admisión a la UCIP y retraso mayor de 4 días para iniciar el tratamiento son condiciones asociadas a mortalidad. ${ }^{14,15}$

Este caso permite reivindicar que, en nuestro medio, la RMSF es una enfermedad circulante, no limitada al área rural o selvática, que puede presentar un curso clínico grave y, por lo tanto, debe considerarse dentro de los diagnósticos diferenciales en pacientes con síndrome exantemático febril y antecedentes epidemiológicos relevantes. Además, su homología con series de casos ya reportadas resalta la necesidad de contar con capacitación continua para los médicos de primer contacto, sobretodo en regiones conurbanas y rurales, en busca de reducir las cifras de mortalidad.

\section{REFERENCIAS}

1. Biggs HM, Behravesh CB, Bradley KK, Dahlgren FS, et al. Diagnosis and management of tickborne rickettsial diseases: rocky mountain spotted fever and other spotted fever group rickettsioses, ehrlichioses, and anaplasmosis United States. MMWR Recomm Rep 2016;65(2):1-44.
2. Buckingham SC, Marshall GS, Schutze GE, Woods CR, et al. Clinical and laboratory features, hospital course, and outcome of Rocky Mountain spotted fever in children. J Pediatr 2007;150(2):180-4, 184 e1.

3. Álvarez-Hernández G, Murillo-Benitez C, Candia-Plata Mdel C, Moro M. Clinical profile and predictors of fatal Rocky Mountain spotted fever in children from Sonora, Mexico. Pediatr Infect Dis J 2015;34(2):125-30.

4. Bacci MR, Namura JJ. Association between sepsis and Rocky Mountain spotted fever. BMJ Case Rep 2012;2012. pii: bcr2012007024

5. Secretaría de Salud, Epidemiología DGAd. Vigilancia epidemiológica de las rickettsiosis. En Manual de procedimientos estandarizados para la vigilancia epidemiológica de las enfermedades transmitidas por vectores. México: Secretaría de Salud; 2012:87-119.

6. Álvarez-Hernández G, Contreras Soto JJ. Letalidad por fiebre manchada por Rickettsia rickettsii en pacientes de un hospital pediátrico del estado de Sonora, 2004-2012. Salud Pública Méx 2013;55(2):151-2.

7. Abarca K, Oteo JA. Aproximación clínica y principales rickettsiosis transmitidas por garrapatas presentes en Latinoamérica. Rev Chil Infectol 2014;31(5):569-76.

8. Zavala-Castro JE, Zavala-Velázquez JE, Walker DH, Ruiz Arcila EE, et al. Fatal human infection with Rickettsia rickettsii, Yucatan, Mexico. Emerg Infect Dis 2006;12(4): 672-4.

9. Jaramillo RHJ, Rodríguez M. Ricketsiosis. Presentación de dos casos, en medio de un brote en Mexicali, Baja California. Med Int Mex 2010;26(2):180-2.

10. Zavala-Velázquez JE, Yu XJ, Walker DH. Unrecognized spotted fever group rickettsiosis masquerading as dengue fever in Mexico. Am J Trop Med Hyg 1996;55(2):157-9.

11. AlvarezG, Rosales C,Sepulveda R. Rocky Mountain spotted fever, a reemerging disease in Arizona and Sonora case study. J Case Reports Stud 2014;2(3):1-3.

12. Eremeeva ME, Dasch GA. Challenges posed by tickborne rickettsiae: eco-epidemiology and public health implications. Front Public Health 2015;3:55.

13. Comer JA, Paddock CD, Childs JE. Urban zoonoses caused by Bartonella, Coxiella, Ehrlichia, and Rickettsia species. Vector Borne Zoonotic Dis 2001;1(2):91-118.

14. Buckingham SC. Tick-borne infections in children: epidemiology, clinical manifestations, and optimal management strategies. Paediatr Drugs 2005;7(3):163-76.

15. Lee N, Ip M, Wong B, Lui G, et al. Risk factors associated with life-threatening rickettsial infections. Am J Trop Med Hyg 2008;78(6):973-8. 